\title{
Electric Dipole Antennas With Magnetic-Coated PEC Cores: Reaching the Chu Lower Bound on Q
}

\author{
Kim, Oleksiy S.
}

Published in:

I E E E Transactions on Antennas and Propagation

Link to article, DOI:

10.1109/TAP.2011.2180331

Publication date:

2012

Document Version

Early version, also known as pre-print

Link back to DTU Orbit

Citation (APA):

Kim, O. S. (2012). Electric Dipole Antennas With Magnetic-Coated PEC Cores: Reaching the Chu Lower Bound on Q. I E E E Transactions on Antennas and Propagation, 60(3), 1616-1619.

https://doi.org/10.1109/TAP.2011.2180331

\section{General rights}

Copyright and moral rights for the publications made accessible in the public portal are retained by the authors and/or other copyright owners and it is a condition of accessing publications that users recognise and abide by the legal requirements associated with these rights.

- Users may download and print one copy of any publication from the public portal for the purpose of private study or research.

- You may not further distribute the material or use it for any profit-making activity or commercial gain

- You may freely distribute the URL identifying the publication in the public portal 


\title{
Electric dipole antennas with magnetic-coated PEC cores: Reaching the Chu lower bound on $\mathrm{Q}$
}

\author{
Oleksiy S. Kim
}

\begin{abstract}
The radiation properties of spherical electric dipole antennas with electric current excitation and material-coated perfectly electrically conducting (PEC) cores are investigated analytically using vector spherical wave functions. Closed-form expressions for electric and magnetic stored energy as well as the radiation quality factor $Q$ are derived. Using these, it is shown that properly selected magnetic coating and radius of the PEC core vastly reduce the internal stored energy, and thus make the $Q$ of an electric dipole antenna approach the Chu lower bound.
\end{abstract}

Index Terms-Electrically small antennas, fundamental limitations, electric dipole, quality factor, Chu lower bound

\section{INTRODUCTION}

A limited frequency bandwidth of electrically small antennas is one of the fundamental problems encountered in antenna miniaturization. It is well known that a fundamental lower bound exists for the antenna radiation quality factor $Q$, which is inversely proportional to the bandwidth for single-mode electrically small antennas. This bound, also known as the Chu lower bound, can be written as [1]-[3]

$$
Q_{\mathrm{Chu}}=\frac{1}{(k a)^{3}}+\frac{1}{k a}
$$

where $k$ is the free-space wave number. The bound (1) is derived assuming no stored energy (except that needed to make an antenna resonant) inside the minimum sphere of radius $a$ circumscribing the antenna. When the internal stored energy is accounted for electric and magnetic dipole antennas with an air-core and electric excitation current, the bounds become $1.5 Q_{\mathrm{Chu}}$ and $3.0 Q_{\mathrm{Chu}}$, respectively, in the limit $k a \rightarrow 0$ [4][6]. Numerous embodiments of both kinds of antenna have confirmed these bounds [7]-[12].

The bounds for the air-core antennas can however be overcome. In [13], [14], it is shown that a solid magnetic core reduces the lower bound for magnetic dipole antennas to

$$
Q \approx\left\{1+0.366(k a)^{2}\right\} Q_{\mathrm{Chu}} \text {. }
$$

Furthermore, a magnetic-coated metal core allows magnetic dipole antennas to reach the Chu lower bound irrespective of their electrical size $\mathrm{ka}$ [15]. Recent investigations of electrically small electric dipole antennas show that the Chu lower bound can be approached for these antennas as well, using thin high-permeability magnetic shells [16], [17].

In this paper, a spherical electric dipole antenna with a material-coated perfectly electrically conducting (PEC) core is studied. In particular, the radiation problem for the electric

O. S. Kim is with the Department of Electrical Engineering, Electromagnetic Systems, Technical University of Denmark, DK-2800 Kgs. Lyngby, Denmark (e-mail: osk@elektro.dtu.dk)

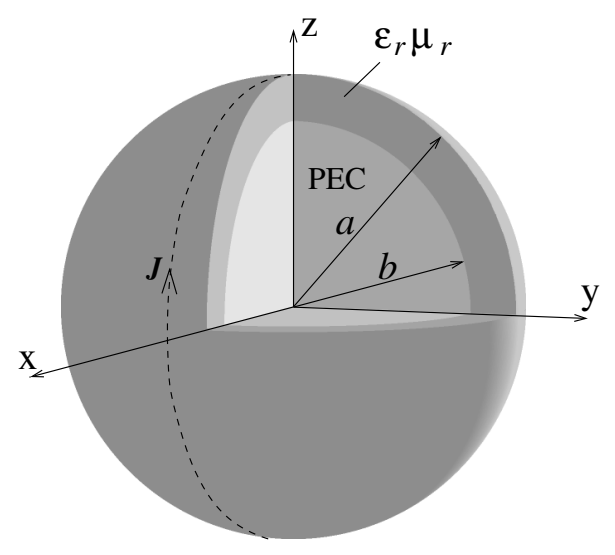

Fig. 1. Electric dipole antenna with a material-coated PEC core. The excitation is a time-harmonic electric surface current density $\mathbf{J}$ distributed over the surface of the antenna.

current excitation is solved rigorously, and expressions for the stored energy and radiation quality factor $Q$ are derived in closed form. It is shown that with such a core an electric dipole antenna can also exhibit the $Q$ approaching the $\mathrm{Chu}$ lower bound.

The time factor $\exp (-i \omega t)$, where $\omega$ denotes the angular frequency and $t$ is the time variable, is assumed and suppressed throughout the paper.

\section{THEORY}

We consider a PEC core of radius $b$ enclosed in a concentric shell of radius $a$ made of a magneto-dielectric material with the relative permittivity $\varepsilon_{\mathrm{r}}$ and relative permeability $\mu_{\mathrm{r}}$ (Fig. 1). The material is assumed to be homogeneous, isotropic, and lossless. A Cartesian coordinate system and an associated spherical coordinate system $(r, \theta, \phi)$ are defined so that their origin is located at the center the antenna core. On the outer surface of the shell, an impressed electric current density radiating the electric dipole $\left(\mathrm{TM}_{10}\right)$ fields is defined as

$$
\mathbf{J}=\hat{\mathbf{a}}_{\theta} J_{0} \sin \theta
$$

where $\hat{\mathbf{a}}_{\theta}$ is the unit vector and $J_{0}$ is the amplitude $(\mathrm{A} / \mathrm{m})$. In practice, the ideal current excitation (3) can be closely approximated in many ways; see, for example, [7], [8], [10], [12].

The electric and magnetic fields inside the shell (superscript "--") and in free space (superscript "+") are represented in terms of vector spherical wave functions $\mathbf{F}(\mathbf{r})[18]$ as

$$
\begin{aligned}
& \mathbf{E}^{-}(\mathbf{r})=\frac{k_{s}}{\sqrt{\eta_{s}}} C^{-} \mathbf{F}_{201}^{(6)}(\mathbf{r}) \\
& \mathbf{H}^{-}(\mathbf{r})=-i k_{s} \sqrt{\eta_{s}} C^{-} \mathbf{F}_{101}^{(6)}(\mathbf{r})
\end{aligned}
$$




$$
\begin{aligned}
& \mathbf{E}^{+}(\mathbf{r})=\frac{k}{\sqrt{\eta}} C^{+} \mathbf{F}_{201}^{(3)}(\mathbf{r}) \\
& \mathbf{H}^{+}(\mathbf{r})=-i k \sqrt{\eta} C^{+} \mathbf{F}_{101}^{(3)}(\mathbf{r})
\end{aligned}
$$

where $\eta_{s}$ and $\eta$ are the intrinsic admittances of the shell and free space, respectively, and $k_{s}=\sqrt{\varepsilon_{\mathrm{r}} \mu_{\mathrm{r}}} k$. The $\mathbf{F}^{(3)}(\mathbf{r})$ functions are as defined in [18] (also provided in [13], [15]). The $\mathbf{F}^{(6)}(\mathbf{r})$ functions can be obtained from, for example, $\mathbf{F}^{(5)}(\mathbf{r})$ in $\left[15\right.$, eq.(5)] by replacing $z_{1}^{(5)}\left(k_{s} r\right)$ with a combination $z_{1}^{(6)}\left(k_{s} r\right)$ of the spherical Bessel $j_{1}$ and Neumann $y_{1}$ functions, so that the boundary condition on the surface of the PEC core is satisfied

$$
\begin{aligned}
z_{1}^{(6)}\left(k_{s} r\right)= & y_{1}\left(k_{s} r\right)\left\{\left(k_{s} b\right) j_{1}\left(k_{s} b\right)\right\}^{\prime} \\
& -j_{1}\left(k_{s} r\right)\left\{\left(k_{s} b\right) y_{1}\left(k_{s} b\right)\right\}^{\prime} .
\end{aligned}
$$

In (5) and from now on, $\{f(x)\}^{\prime}$ denotes the derivative of the function $f(x)$ with respect to its argument $x$.

The coefficients $C^{ \pm}$are determined from the boundary condition on the outer surface of the shell $r=a$, subject to (3), as

$$
\begin{aligned}
& C^{-}=J_{0} i a \frac{1}{\sqrt{\eta_{s}}} \frac{4 \sqrt{\pi}}{\sqrt{6}}\left\{(k a) h_{1}^{(1)}(k a)\right\}^{\prime} I^{-1} \\
& C^{+}=J_{0} i a \frac{\sqrt{\eta}}{\eta_{s}} \frac{4 \sqrt{\pi}}{\sqrt{6}}\left\{\left(k_{s} a\right) z_{1}^{(6)}\left(k_{s} a\right)\right\}^{\prime} I^{-1}
\end{aligned}
$$

where

$$
\begin{aligned}
I= & \frac{\eta}{\eta_{s}} k a h_{1}^{(1)}(k a)\left\{\left(k_{s} a\right) z_{1}^{(6)}\left(k_{s} a\right)\right\}^{\prime} \\
& -k_{s} a z_{1}^{(6)}\left(k_{s} a\right)\left\{(k a) h_{1}^{(1)}(k a)\right\}^{\prime} .
\end{aligned}
$$

To determine the stored energy, the energy density of the propagating field in free space is subtracted from the total energy density [3]. Then, by a direct spatial integration the internal electric $W_{\mathrm{E}}^{-}$, internal magnetic $W_{\mathrm{H}}^{-}$, external electric $W_{\mathrm{E}}^{+}$, and external magnetic $W_{\mathrm{H}}^{+}$stored energy are obtained as

$$
\begin{aligned}
& W_{\mathrm{E} / \mathrm{H}}^{ \pm}=\frac{1}{4 \omega}\left|C^{ \pm}\right|^{2} \hat{W}_{\mathrm{E} / \mathrm{H}}^{ \pm} \quad \text { with } \\
& \hat{W}_{\mathrm{E}}^{-}=\frac{1}{2}\left\{L\left(k_{s} a\right)-L\left(k_{s} b\right)+\left(k_{s} a\right)\left\{R_{1}\left(k_{s} a\right)\right\}^{\prime 2}\right\} \\
& \hat{W}_{\mathrm{H}}^{-}=\frac{1}{2}\left\{L\left(k_{s} a\right)-L\left(k_{s} b\right)-\left(k_{s} a\right) R_{2}\left(k_{s} a\right)\left\{R_{1}\left(k_{s} a\right)\right\}^{\prime}\right\} \\
& \hat{W}_{\mathrm{E}}^{+}=\frac{1}{(k a)^{3}}+\frac{1}{k a} \\
& \hat{W}_{\mathrm{H}}^{+}=\frac{1}{k a}
\end{aligned}
$$

where

$$
\begin{aligned}
L\left(k_{s} r\right)= & R_{1}\left(k_{s} r\right)\left[\left(k_{s} r\right) R_{1}\left(k_{s} r\right)-R_{2}\left(k_{s} r\right)\right] \\
R_{l}\left(k_{s} r\right)= & \left(k_{s} r\right) y_{l}\left(k_{s} r\right)\left\{\left(k_{s} b\right) j_{1}\left(k_{s} b\right)\right\}^{\prime} \\
& -\left(k_{s} r\right) j_{l}\left(k_{s} r\right)\left\{\left(k_{s} b\right) y_{1}\left(k_{s} b\right)\right\}^{\prime} .
\end{aligned}
$$

Finally, the radiation quality factor $Q$ is found as

$$
\begin{aligned}
Q & =2 \omega \frac{\max \left(W_{\mathrm{E}}^{-}+W_{\mathrm{E}}^{+}, W_{\mathrm{H}}^{-}+W_{\mathrm{H}}^{+}\right)}{P_{\mathrm{rad}}} \\
& =\max \left(\frac{\left|C^{-}\right|^{2}}{\left|C^{+}\right|^{2}} \hat{W}_{\mathrm{E}}^{-}+\hat{W}_{\mathrm{E}}^{+}, \frac{\left|C^{-}\right|^{2}}{\left|C^{+}\right|^{2}} \hat{W}_{\mathrm{H}}^{-}+\hat{W}_{\mathrm{H}}^{+}\right)
\end{aligned}
$$

using (6), (7), and the radiated power $P_{\mathrm{rad}}=\frac{1}{2}\left|C^{+}\right|^{2}$.

\section{Minimizing $Q$}

First, it is instructive to examine the characteristics of the antenna with a pure magnetic shell $\left(\varepsilon_{\mathrm{r}}=1\right)$ and a fixed electrical size $k a$. The ratio of the magnetic $W_{\mathrm{H}}=W_{\mathrm{H}}^{+}+W_{\mathrm{H}}^{-}$ to electric $W_{\mathrm{E}}=W_{\mathrm{E}}^{+}+W_{\mathrm{E}}^{-}$stored energy and the quality factor normalized to the Chu lower bound $Q / Q_{\mathrm{Chu}}$ are shown in Fig. $2 \mathrm{a}$ and $2 \mathrm{~b}$, respectively, versus the shell permeability $\mu_{\mathrm{r}}$ for $k a=0.5$ and three values of the relative radius of the PEC core $b / a=0.0,0.6$, and 0.9. For the antenna with $b / a=0$, the stored energy is predominantly electric, as it should be for an electrically small electric dipole antenna, in which the stored magnetic energy is further suppressed by a solid magnetic core. The magnetic stored energy dominates only in very narrow regions between internal and radiating resonances, which appear interchangeably as $\mu_{\mathrm{r}}$ increases (Fig. 2). The first type of resonance differs from the second one by the absence of the radiated power, so that $Q \rightarrow \infty$. As the relative radius of the PEC core increases, the separation between resonances becomes larger - particularly, for the first pair of resonances - and in these regions the magnetic stored energy dominates. An important consequence of the increased separation between the resonances is that the radiating resonance moves away from the region, where the $Q$ is very high due to the internal resonance (Fig. 2b). As we show later, this opens a possibility for a self-resonant pure electric dipole antenna with a $Q$ close to the Chu lower bound.

Meanwhile, we focus on determining the optimal geometry and material parameters minimizing the antenna $Q$. Inspecting Fig. 2b, we note that the increasing $b / a$ leads to higher $Q$ in the air-core case $^{1}\left(\mu_{\mathrm{r}}=1\right)$, whereas the minima between the resonances becomes deeper. Obviously, there is an optimum permeability $\mu_{\mathrm{r}}$ providing the lowest $Q$ for given $k a, b / a$, and $\varepsilon_{\mathrm{r}}$. On the other hand, if both material parameters $\left(\mu_{\mathrm{r}}\right.$ and $\varepsilon_{\mathrm{r}}$ ) are fixed, an optimum $b / a$ can be determined for a given $k a$. Here, we consider the latter case, because it seems somewhat more practical to design geometrical rather than material parameters of an antenna.

The optimum radius of the PEC core is obtained by taking the derivative of (8) with respect to $b / a$ as

$$
\begin{aligned}
& \frac{\partial Q}{\partial(b / a)}=\varepsilon_{\mathrm{r}} \frac{1-(k a)^{2}+(k a)^{4}}{(k a)^{3}}\left\{\begin{array}{l}
\left.\frac{\partial}{\partial(b / a)}\left\{\begin{array}{l}
\frac{\hat{W}_{\mathrm{E}}^{-}}{\left(k_{s} a\right)\left\{R_{1}\left(k_{s} a\right)\right\}^{\prime 2}} \\
\frac{\partial}{\partial(b / a)}
\end{array}\right\} \frac{\hat{W}_{\mathrm{H}}^{-}}{\left(k_{s} a\right)\left\{R_{1}\left(k_{s} a\right)\right\}^{\prime 2}}\right\}
\end{array}\right. \\
& \equiv \varepsilon_{\mathrm{r}} f(k a) \begin{cases}\frac{\partial \Psi_{\mathrm{E}}\left(k_{s} a, b / a\right)}{\partial(b / a)} & \text { for } W_{\mathrm{E}}>W_{\mathrm{H}} \\
\frac{\partial \Psi_{\mathrm{H}}\left(k_{s} a, b / a\right)}{\partial(b / a)} & \text { for } W_{\mathrm{E}}<W_{\mathrm{H}}\end{cases}
\end{aligned}
$$

and then setting the result equal to zero

$$
0= \begin{cases}\frac{\partial \Psi_{\mathrm{E}}\left(k_{s} a, b / a\right)}{\partial(b / a)} & \text { for } W_{\mathrm{E}}>W_{\mathrm{H}} \\ \frac{\partial \Psi_{\mathrm{H}}\left(k_{s} a, b / a\right)}{\partial(b / a)} & \text { for } W_{\mathrm{E}}<W_{\mathrm{H}}\end{cases}
$$

The equation (10) has multiple solutions; one with the highest $b / a$ provides the lowest $Q$ and is denoted as

$$
b / a= \begin{cases}F_{\mathrm{E}}\left(k_{s} a\right) & \text { for } W_{\mathrm{E}}>W_{\mathrm{H}} \\ F_{\mathrm{H}}\left(k_{s} a\right) & \text { for } W_{\mathrm{E}}<W_{\mathrm{H}}\end{cases}
$$

\footnotetext{
${ }^{1}$ The reason for this behaviour is the same as for the $\mathrm{TE}_{10}$-mode case, see $[15$, Section II.C]
} 


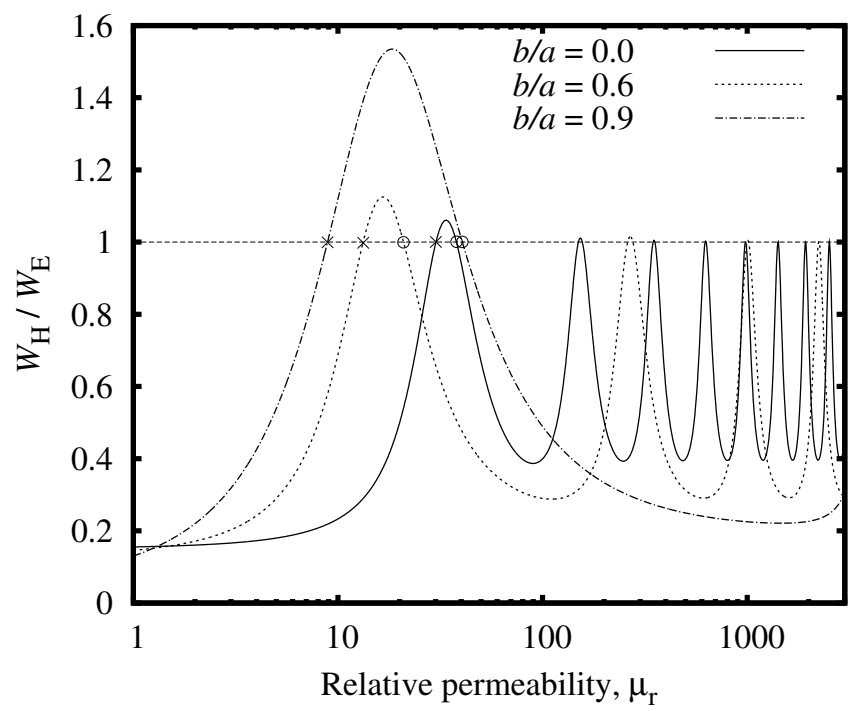

(a)

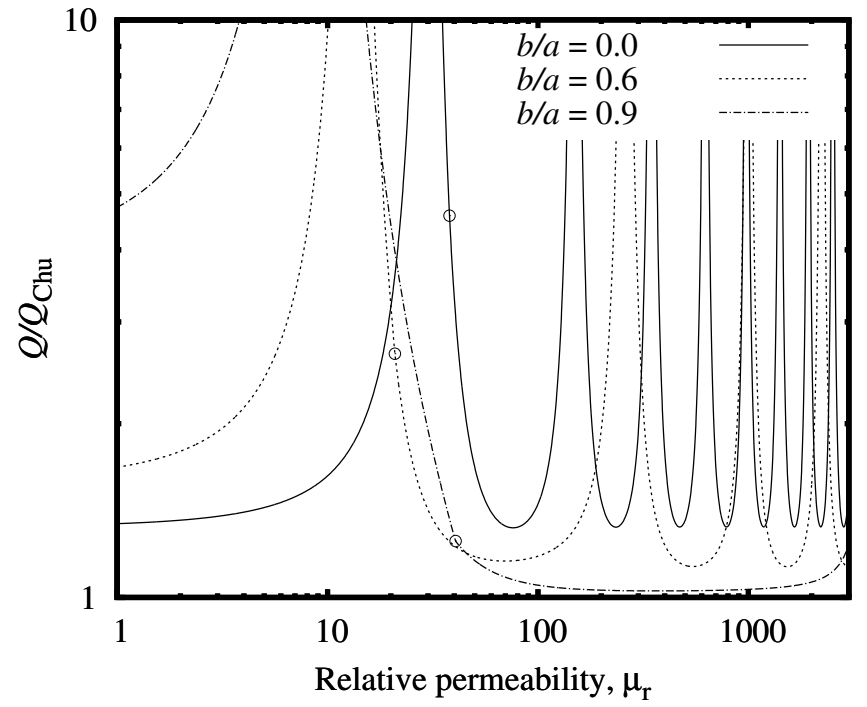

(b)

Fig. 2. Stored energy and $Q$ as functions of $\mu_{\mathrm{r}}\left(\varepsilon_{\mathrm{r}}=1\right)$ for fixed $k a=0.5$ : (a) Ratio of magnetic to electric stored energy; (b) $Q$ normalized to the Chu lower bound. The first internal and radiating resonances are marked with ' $x$ ' and 'o', respectively.

In other words, the function $b / a=F_{\mathrm{E}}\left(k_{s} a\right)$ is the solution of (10a) and the function $b / a=F_{\mathrm{H}}\left(k_{s} a\right)$ is the solution of (10b). Figure 3a shows both functions along with the parameters for the first radiating resonance. A resonant curve for given $k a$ and $\varepsilon_{\mathrm{r}}$ divides the plot into two regions. The region to the left of the resonant curve corresponds to $W_{\mathrm{E}}<W_{\mathrm{H}}$, whereas to the right $W_{\mathrm{E}}>W_{\mathrm{H}}$. Consequently, the solution of (10) first follows the $F_{\mathrm{H}}\left(k_{s} a\right)$ curve; once the resonance is reached, the solution transits to the $F_{\mathrm{E}}\left(k_{s} a\right)$ curve. In the transition region, no solution exists for the equation (10), and therefore the transition between $F_{\mathrm{H}}\left(k_{s} a\right)$ and $F_{\mathrm{E}}\left(k_{s} a\right)$ occurs along the resonant curve. For some values of $k a$ and $\varepsilon_{\mathrm{r}}$, the resonant curve lies further to the right of the $F_{\mathrm{E}}\left(k_{s} a\right)$ curve, as, for example, for $k a=1.5$ and $\varepsilon_{\mathrm{r}}=1$ in Fig. 3a. In this case, the lowest $Q$ corresponds to the parameters that lie not at the $F_{\mathrm{E}}\left(k_{s} a\right)$ curve, but along the resonant curve instead.

Note, that both $F_{\mathrm{H}}\left(k_{s} a\right)$ and $F_{\mathrm{E}}\left(k_{s} a\right)$ start at $k_{s} a \approx 1.416$.

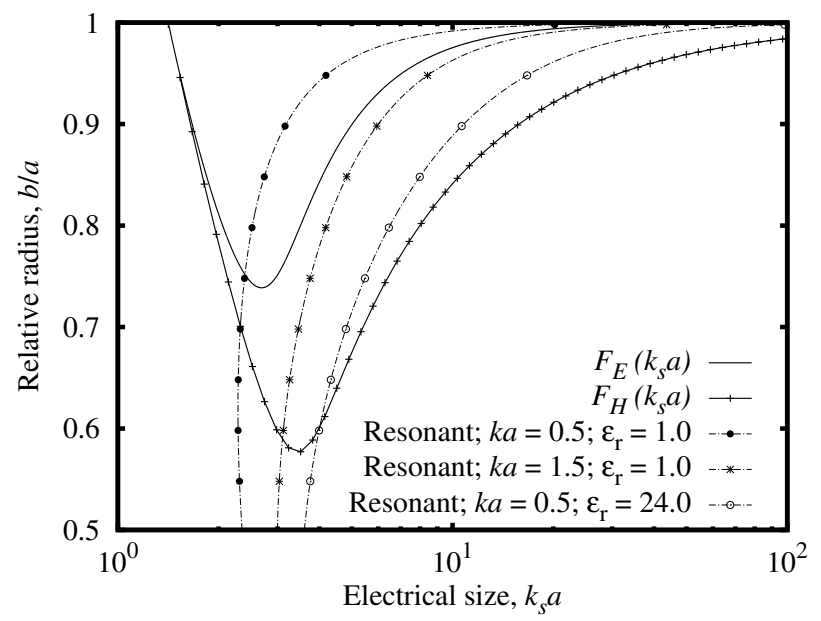

(a)

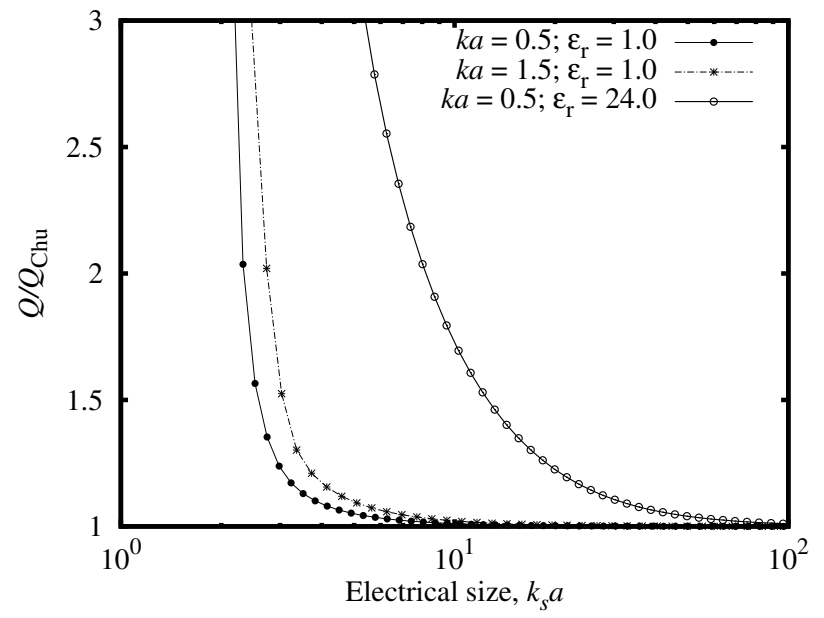

(b)

Fig. 3. Optimum and resonant relative radius of the PEC core $b / a$ (a) and the corresponding lowest achievable ratio $Q / Q_{\mathrm{Chu}}$ for the optimum $b / a$ (b).

Below this value, a solid core $(b / a=0)$ should be used. If an air-core is also an option, it is preferable to a magnetic-coated PEC core with parameters in the region $W_{\mathrm{E}}<W_{\mathrm{H}}$ in Fig. 3a, where the $Q$ is generally larger than $1.5 Q_{\text {Chu }}$.

By substituting the (11) into (8), an expression for the lowest possible $Q$ corresponding to the optimum $b / a=F_{\mathrm{E} / \mathrm{H}}\left(k_{s} a\right)$ is obtained as

$Q= \begin{cases}\varepsilon_{\mathrm{r}} f(k a) \Psi_{\mathrm{E}}\left(k_{s} a, F_{\mathrm{E}}\left(k_{s} a\right)\right)+Q_{\mathrm{Chu}} & \text { for } W_{\mathrm{E}}>W_{\mathrm{H}} \\ \varepsilon_{\mathrm{r}} f(k a) \Psi_{\mathrm{H}}\left(k_{s} a, F_{\mathrm{H}}\left(k_{s} a\right)\right)+\frac{1}{k a} & \text { for } W_{\mathrm{E}}<W_{\mathrm{H}}\end{cases}$

The resulting $Q$ is plotted in Fig. 3b. From (12) and Fig. 3b, it is clear that the higher permittivity results in the higher $Q$. Nevertheless, as $\mu_{\mathrm{r}}-$ and thus $k_{s} a-$ increases, $b / a \rightarrow 1$ and the $Q$ approaches the Chu lower bound $Q_{\text {Chu }}$ irrespective of the antenna electrical size $k a$ and permittivity of the shell $\varepsilon_{\mathrm{r}}$.

As discussed above, for some antenna sizes $k a$ and shell permittivity $\varepsilon_{\mathrm{r}}$, the lowest $Q$ is achieved at the resonance. However, for electrically small antennas $(k a<0.5)$ and low permittivity, the optimal parameters for the lowest possible $Q$ result in an antenna with the dominating electric stored energy (Fig. 3a). On the other hand, as seen in Fig. 3a, for large values of permeability $\mu_{\mathrm{r}}$, the resonant curve and the optimum $b / a=F_{\mathrm{E}}\left(k_{s} a\right)$ tend to each other, which implies 


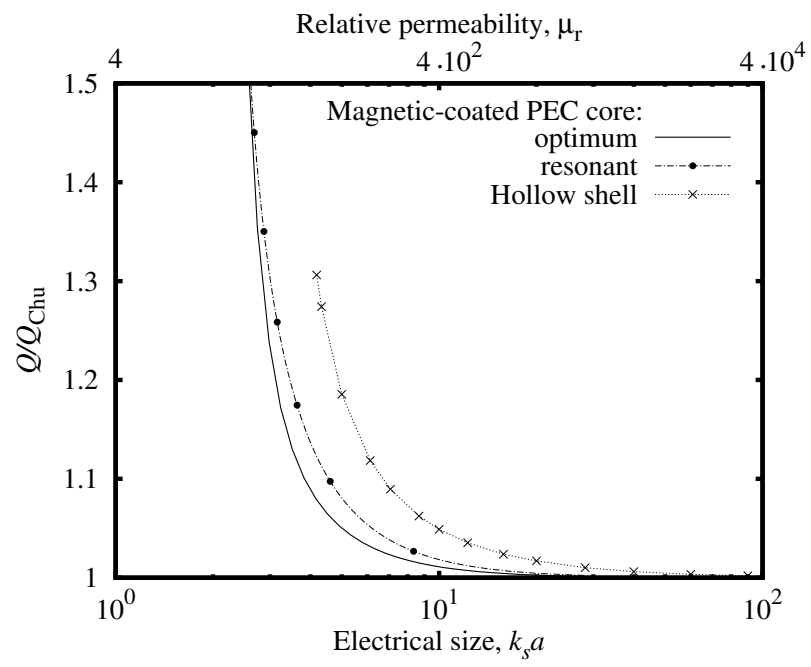

Fig. 4. The lowest achievable ratio $Q / Q_{\mathrm{Chu}}$ for a magnetic-coated PEC core (for the optimum and resonant $b / a$ ) and a hollow magnetic shell. In all cases, $k a=0.5$ and $\varepsilon_{\mathrm{r}}=1$.

that a self-resonant electric dipole antenna can be designed without significant sacrifice in $Q$. For example, for $k a=0.5$ and $\varepsilon_{\mathrm{r}}=1$, the difference between the $Q$ 's corresponding to the optimum and resonant $b / a$ becomes practically negligible for $\mu_{\mathrm{r}}>1000\left(k_{s} a>15.8\right)$, as shown in Fig. 4 .

The self-resonance of the $\mathrm{TM}_{10}$ mode is an advantage for an electric dipole antenna, since no additional reactive components are needed to compensate for the input reactance. On the other hand, if the lowest possible $Q$ is a primary goal, a dualmode $\mathrm{TM}_{10}+\mathrm{TE}_{10}$ antenna should be considered [1]. In such an antenna, the $\mathrm{TM}_{10}$ mode should generate an excess electric stored energy to match a corresponding amount of the excess magnetic stored energy of the $\mathrm{TE}_{10}$ mode, in which case the $Q$ close to $0.5 Q_{\text {Chu }}$ can be achieved, as demonstrated in [19].

A configuration with a hollow magnetic shell (without the PEC core) and the excitation electric current on the outer surface has also been investigated. COMSOL's 2D RF module for bodies of revolution was used. The antenna of $k a=0.5$ was simulated for a number of $\mu_{\mathrm{r}}$ values of the shell $\left(\varepsilon_{\mathrm{r}}=1\right)$; for each $\mu_{\mathrm{r}}$, the lowest $Q$ in the range of $b / a$ ( $b$ is the inner radius of the shell, in this case) from 0 to 1 was identified, and the results are presented in Fig. 4. In the limit of infinitely high permeability, the $Q$ for both configurations - with and without PEC core - approaches the Chu lower bound. However, for finite $\mu_{\mathrm{r}}$, the PEC core allows the coating to have significantly lower permeability $\mu_{\mathrm{r}}$, as compared to the hollow shell, to achieve the same value of $Q / Q_{\mathrm{Chu}}$. The reason is that a thin PEC-backed layer of magnetic material better approximates the perfect magnetic conductor. For instance, the hollow shell needs to have $\mu_{\mathrm{r}} \approx 390$ to yield $Q / Q_{\mathrm{Chu}}=1.05$, whereas the magnetic coating on the PEC core requires only $\mu_{\mathrm{r}} \approx 100$ to reach the same $Q / Q_{\mathrm{Chu}}=1.05$.

\section{CONCLUSION}

The radiation problem for the $\mathrm{TM}_{10}$ electric current on the surface of a material-coated spherical PEC core is solved analytically using vector spherical wave functions. Closedform expressions for the electric and magnetic stored energy as well as for the radiation quality factor $Q$ are presented. The optimum relative radius of the PEC core $b / a$ providing the lowest possible $Q$ for a given antenna electrical size $k a$ and material parameters $\varepsilon_{\mathrm{r}}$ and $\mu_{\mathrm{r}}$ of the coating is found. It is shown that a PEC core with an electrically thin high-permeability magnetic coating significantly reduces the internal stored energy, and thus the $Q$ of the electric dipole antenna closely approaches the Chu lower bound. For instance, an antenna of the size $k a=0.5$ with the relative radius of the PEC core $b / a=0.975$ and a pure magnetic coating with $\mu_{\mathrm{r}}=400$ yields the quality factor $Q=1.01 Q_{\mathrm{Chu}}$.

\section{REFERENCES}

[1] L. J. Chu, "Physical limitations of omni-directional antennas," J. Appl. Phys., vol. 19, no. 12, pp. 1163-1175, 1948.

[2] R. Collin and S. Rothschild, "Evaluation of antenna Q," IEEE Trans. Antennas Propagat., vol. 12, no. 1, pp. 23-27, Jan. 1964.

[3] J. S. McLean, "A re-examination of the fundamental limits on the radiation Q of electrically small antennas," IEEE Trans. Antennas Propagat., vol. 44, no. 5, pp. 672-676, May 1996.

[4] H. A. Wheeler, "The spherical coil as an inductor, shield, or antenna," Proc. IRE, vol. 46, no. 9, pp. 1595-1602, 1958.

[5] H. L. Thal, "New radiation Q limits for spherical wire antennas," IEEE Trans. Antennas Propagat., vol. 54, no. 10, pp. 2757-2763, Oct. 2006.

[6] R. Hansen and R. Collin, "A new Chu formula for Q," IEEE Antennas Propagat. Mag., vol. 51, no. 5, pp. 38-41, 2009.

[7] S. R. Best, "The radiation properties of electrically small folded spherical helix antennas," IEEE Trans. Antennas Propagat., vol. 52, no. 4, pp. 953-960, Apr. 2004.

[8] — , "Low Q electrically small linear and elliptical polarized spherical dipole antennas," IEEE Trans. Antennas Propagat., vol. 53, no. 3, pp. 1047-1053, Mar. 2005.

[9] — , "A low Q electrically small magnetic (TE mode) dipole," IEEE Antennas Wireless Propagat. Lett., vol. 8, pp. 572-575, 2009.

[10] H. R. Stuart, H. R. Stuart, and C. Tran, "Small spherical antennas using arrays of electromagnetically coupled planar elements," IEEE Antennas and Wireless Propagat. Lett., vol. 6, pp. 7-10, 2007.

[11] O. S. Kim, "Low-Q electrically small spherical magnetic dipole antennas," IEEE Trans. Antennas Propagat., vol. 58, no. 7, pp. 2210-2217, July 2010.

[12] — "Novel electrically small spherical electric dipole antenna," in Proc. of International Workshop on Antenna Technology (iWAT2010), Lisbon, Portugal, 2010.

[13] O. S. Kim, O. Breinbjerg, and A. D. Yaghjian, "Electrically small magnetic dipole antennas with quality factors approaching the Chu lower bound," IEEE Trans. Antennas Propagat., vol. 58, no. 6, pp. 1898-1906, June 2010.

[14] O. S. Kim and O. Breinbjerg, "Lower bound for the radiation Q of electrically small magnetic dipole antennas with solid magnetodielectric core," IEEE Trans. Antennas Propagat., vol. 59, no. 2, pp. 679-681, February 2011.

[15] — "Reaching the Chu lower bound on $\mathrm{Q}$ with magnetic dipole antennas using a magnetic-coated PEC core," IEEE Trans. Antennas Propagat., vol. 59, no. 8, pp. 2799-2805, August 2011.

[16] A. D. Yaghjian and H. R. Stuart, "Lower bounds on the Q of electrically small dipole antennas," IEEE Trans. Antennas Propagat., vol. 58, no. 10, pp. 3114-3121, November 2010 .

[17] H. R. Stuart and A. D. Yaghjian, "Approaching the lower bounds on $\mathrm{Q}$ for electrically small electric-dipole antennas using high permeability shells," IEEE Trans. Antennas Propagat., vol. 58, no. 12, pp. 3865-3872, December 2010.

[18] J. E. Hansen, Spherical Near-Field Antenna Measurements. London, U.K.: Peter Peregrinus, 1988.

[19] O. S. Kim, "Minimum Q circularly polarized electrically small spherical antenna," in Proc. IEEE Antennas Propag. Soc. Int. Symp., Spokane, Washington, USA, July 3-8 2011, pp. 750-752. 